\title{
Effect of Active Dynamic Versus Passive Static Stretching on Hamstring Muscle Tightness in Healthy Female Students: A Randomized Trial Study
}

\author{
Mojtaba Heshmatipour ${ }^{1}$, Azam Esfandiari ${ }^{1}$, Maryam Kazemi Naeini ${ }^{2}$, Mehdi Raei ${ }^{3,4^{*}}$, Omolbanin \\ Firoozpur ${ }^{1}$, Neda Shariatinia ${ }^{1}$, Kiavash Hushmandi ${ }^{5}$
}

\begin{abstract}
'Department of Physical Therapy, Faculty of Rehabilitation Sciences, Isfahan University of Medical Sciences, Isfahan, Iran

${ }^{2}$ Student Research Committee, School of Health, Isfahan University of Medical Sciences, Isfahan, Iran

${ }^{3}$ Baqiyatallah Research Center for Gastroenterology and Liver Diseases (BRCGL), Baqiyatallah University of Medical Sciences, Tehran, Iran

${ }^{4}$ Department of Epidemiology and Biostatistics, Faculty of Health, Baqiyatallah University of Medical Sciences, Tehran, Iran

${ }^{5}$ Faculty of Veterinary Medicine, University of Tehran, Tehran, Iran
\end{abstract}

*Corresponding Author: Mehdi Raei, Ph.D., Assistant Professor, Baqiyatallah Research Center for Gastroenterology and Liver Diseases (BRCGL), Baqiyatallah University of Medical Sciences, Tehran, Iran. Fax: +98-2188069126, Tel:

+98-9122473070, Email: Mehdi_r_d@yahoo.com

Received June 15, 2019; Accepted November 8, 2019; Online Published December 15, 2019

\begin{abstract}
Background: For decades, static stretching has been the standard benchmark for training programs, because it has been shown to increase flexibility compared with other methods of stretching.

Objective: The current study investigated and compared the effects of active dynamic stretching and passive static stretching on hamstring tightness.

Methods: For this experiment, 64 female students were enrolled and randomly assigned to active dynamic or passive static stretching groups $(n=32$ each). The first and second experimental groups were trained with repetitive dynamic stretching and static stretching exercises, respectively. Exercises were performed 10 times per limb, 3 times per day, 5 days per week for 4 weeks. Hamstring muscle length measurements were repeated in weeks 2 and 4 . Statistical analysis of the results was performed by t-test and repeated measures ANOVA using SPSS 15.

Results: Both experimental groups showed significant improvements in the active knee extension range of motion during the intervention $(P<0.001)$. However, active stretching showed better results and had a greater effect on range of motion in comparison with static stretching.

Conclusion: Active dynamic training can be considered a suitable method for increasing the flexibility of the hamstring muscle and, consequently, reducing the complications and problems associated with hamstring tightness.

Keywords: Training, Exercise, Therapeutic, Treatment
\end{abstract}

\section{Background}

The hamstring muscle is one of the crucial muscles of the lower extremities. A part of the tonic muscle group, this muscle consists of 3 two-joint muscles (TJM), an extensor of the leg joint, and a knee joint flexor. Flexibility in TJMs is of particular importance. The flexibility of the hamstring muscle group is essential in knee extension and for many daily activities in which the muscles mentioned above simultaneously extend over the 2 hip and knee joints. In the absence of proper flexibility, muscle function is impaired. ${ }^{1-3}$

Hamstring tightness is common for many different reasons. ${ }^{1}$ Naturally, the muscles are inactive at a shorter length, and consequently, their inactiveness has an effect on other musculoskeletal systems in the area, ${ }^{4}$ causing problems such as reduced function, increased chance of muscle damage, back pain, and biomechanical changes in the joints of the region. ${ }^{5}$

Given that muscles tend to be tight in dysfunction and also because of the habit of sitting on 2 knees (known as Turkish style) in middle eastern nations, especially Iran, the prevalence of hamstring tightness is explicable. ${ }^{6}$

For decades, static stretching has been used as the standard benchmark for training programs, because it

Copyright $\odot 2019$ The Author(s). This is an open-access article distributed under the terms of the Creative Commons Attribution License (http:// creativecommons.org/licenses/by/4.0), which permits unrestricted use, distribution, and reproduction in any medium, provided the original work is properly cited. 
has been shown to increase flexibility in contrast to other methods of stretching. ${ }^{7}$

More recently, studies have considered dynamic stretching. In a study conducted by Ayala et al in $2013^{8}$ which compared active stretching in healthy men and in those with hamstring flexibility limitation, it was shown that active stretching had a positive effect on both groups. In 2015, Rani et $\mathrm{al}^{9}$ compared 2 active stretching techniques on hamstring flexibility in asymptomatic individuals; they used a modified hold-relax technique of proprioceptive neuromuscular facilitation (PNF) and neural mobilization for improving the flexibility of hamstrings on male subjects. The results of their study showed that both hold-relax and neural mobilization are equally effective in improving hamstring flexibility.

In 2016, Ahmed and Samhan ${ }^{10}$ compared the short-term effects of neurodynamic and static stretching techniques on hamstring flexibility in healthy male subjects and showed that neurodynamic stretching was more effective than static stretching.

\section{Objective}

Considering the fairly high prevalence of hamstring tightness in the general population (between 30\% and $50 \%$ ), the existence of various theories for the treatment of this complication, and the lack of responsiveness to these methods, ${ }^{11-13}$ the need for a comparative research between conventional methods such as "passive static stretching" and the proposed method of "active dynamic stretching" was realized.

\section{Methods}

\subsection{Study Design}

A single-blind, randomized clinical trial was done to assess and compare the effects of active dynamic stretching and passive static stretching on hamstring tightness.

\subsection{Subjects}

Female students (due to their availability and cooperation) aged 18 to 30 years residing in the dormitories of Isfahan University of Medical Sciences were included in the study. A total of 109 people from this age group were evaluated for hamstring muscle length. Subjects were considered eligible if they had no history of any knee, hip, or spinal conditions that necessitated medical intervention or any injury or disease expected to affect hamstring length or ability to perform the exercises.

The sample size was estimated using the general formula for comparison of 2 means as follows. Considering $\alpha=0.05$, $\beta=0.2$ (confidence interval $=0.95$ and power $=80 \%$ ), the hypothesized difference between means (considered to be significant) equaled 3.5; considering the hypothesized standard deviation in the first and second group $(\mathrm{S} 1=\mathrm{S} 2=5)$, the sample size was 32 in each group.

$$
\mathrm{n}=\frac{\left(\mathrm{z}_{1-\alpha / 2}+\mathrm{z}_{1-\beta}\right)^{2}\left(\mathrm{~s}_{1}^{2}+\mathrm{s}_{2}^{2}\right)}{\mathrm{d}^{2}}=32
$$

Healthy girls aged 18 to 30 years who had hamstring tightness were enrolled in this study regardless of height and weight. Of the total 82 subjects approached, 64 people were willing to cooperate in this study.

\subsection{Procedure}

In the first stage, the research and its aims were briefly described for the students. After ensuring the students' willingness to participate, the initial active knee extension test $(\mathrm{AKE})^{14}$ was performed.

The subjects were randomly divided into 2 experimental groups of 2 different types of exercises. This study was of a randomized block design (balanced random block) with the 4-block method. The first experimental group included 32 female students who were given active dynamic stretch exercises. The second experimental group included about 32 female students between 18 and 30 years of age who were given passive static stretch training. The training method was presented to the subjects in written form with illustrations of the exercises. To ensure proper performance of the stretching methods, subjects performed their first stretching exercises with the examiner. Two and 4 weeks after the beginning of the training, measurements were taken by the same examiners who performed the initial test, and the results were recorded by the third examiner at each measurement step.

\subsection{Measurement Protocol}

Pursuant to other researchers who have selected AKE as an excellent objective test with high repeatability for measuring hamstring muscle length, ${ }^{14,15}$ this study also employed the AKE test. Based on the theory of Webright et $\mathrm{al}^{16}$ the natural muscle length was considered to be 15 degrees or less than the total extension of the knee.

\subsection{Reliability}

Prior to the study, a preliminary study was conducted to obtain intra-rater reliability. At this stage, 15 female students from Isfahan University of Medical Sciences were selected based on the exclusion criteria, and they expressed their willingness to participate in the test before the preliminary study.

To determine the repeatability of measuring hamstring muscle length by the AKE test with a goniometer (Laserliner, Germany), each measurement was performed in 2 stages 30 minutes apart ${ }^{17}$ on the left limb of the subjects, and the intra-rater reliability was obtained.

Relative repeatability was determined by evaluating the correlation between the values obtained in the measurements. As a result, when the correlation between the values obtained at each measurement was closer to 1 , the repeatability was higher.

The correlation test was used to calculate the ICC. Absolute repeatability was determined by comparing the mean of the obtained values in 2 measurements. After obtaining the results and ensuring the reliability of the measurements, the absolute and relative measurements of 
the test were performed on the main samples.

\subsection{Treatment Protocol/Stretching Procedure}

3.6.1. Method of Active Dynamic Stretch Exercise

To begin the stretching, subjects were in the AKE test position. At knee extension, the subject held a position which provoked a tremor in the leg for 20 seconds, then abandoned it. Subjects then repeated the same procedure on the opposite limb. ${ }^{9,18-20}$ This exercise was performed 10 times per limb, 3 times per day, 5 days per week for 4 weeks.

\subsection{Passive Static Stretching Exercise}

This exercise was performed using the supine hamstring stretch method. ${ }^{21}$ Subjects sat on a hard surface with both hip joints in the $90^{\circ}$-flexed position and the leg extended from the knee. Subjects put a towel on the foot and pulled it toward the chest with both hands, keeping the knee flat and fingers in the dorsi-flexion position. The stretch was maintained for 20 seconds, ${ }^{22}$ and then the leg was returned to the original state. The same exercise was repeated for the other leg. The frequency and duration of the exercise were similar to those of the dynamic active stretching exercise.

\subsection{Data Analyses}

Data on knee extension rate was recorded in initial stages and after the exercise. Data was analyzed using SPSS software (version 15), and the results were analyzed using ANOVA repeated measures test and the independent $t$ test.

\section{Results}

Participants in the 2 groups were analyzed for characteristics such as age $(24.49 \pm 3.11$ vs. $23.65 \pm 2.87)$ and body mass index $(23.57 \pm 2.54$ vs. $22.85 \pm 2.13)$, and no statistically significant difference was seen.

In this study, people with $\mathrm{AKE}$ above $15^{\circ}$ were considered to have a tight hamstring. Of the 109 people who were evaluated, 82 had tightness in these muscles. Therefore, the frequency of hamstring muscle tightness in the studied students was $75 \%$.
To find the relative and absolute repeatability of the AKE test, the method of Youdas ${ }^{23}$ was used, which considers intraclass correlation (ICC) $>90$ as an excellent repeatability. In this study, excellent repeatability of the AKE test was obtained (ICC $=0.99$ ).

Thirty-two girls were enrolled in each exercise group. There was no significant difference in mean knee extension angle at the beginning of the study between the 2 groups. However, a significant difference was seen in the second and fourth weeks. After performing active dynamic or passive static exercises in the given treatment period, the mean knee extension angle was significantly decreased in both groups, and a significantly greater reduction was seen in the active dynamic group $(P<0.001)$ than in the passive static group (Table 1).

The comparison of angle differences in the beginning and at the second week and in the beginning and fourth week in both groups showed a greater difference in the active dynamic group than in the passive static group (Table 2).

\section{Discussion}

The frequency of hamstring muscle tightness in female students in this study was $75 \%$. The mean reduction in knee extension angle was significantly greater in the active dynamic group compared with the passive static group. The difference in the initial angle and the angle at the second week and in the fourth week was greater in the active dynamic group than in the passive static group.

The relatively high frequency of hamstring muscle tightness, ${ }^{11-13,24,25}$ the complications associated with this condition, and the low responsiveness to therapies commonly used to increase hamstring flexibility necessitate more extensive investigations into therapy methods.

According to Donatelli et $\mathrm{al}^{26}$ and Travell et $\mathrm{al}^{3}$ shortness of hamstring muscles causes various complications such as insufficiency and muscle inactivity ${ }^{4}$ and affects the musculoskeletal system. Other problems associated with this condition include increased risk of muscular

Table 1. The Process of Changing the Angle of Extension in Both Legs in 2 Groups

\begin{tabular}{lcc}
\hline \multirow{2}{*}{ Time } & \multicolumn{2}{c}{ Mean \pm Standard Deviation } \\
\cline { 2 - 3 } & P Value & Passive Static Group \\
\hline Initial angle of both legs & $31.25 \pm 8.89$ & $29.39 \pm 7.51$ \\
Angle of both legs after 2 weeks & $23.87 \pm 6.06$ & $26.64 \pm 5.39$ \\
Angle of both legs after 4 weeks & $22.00 \pm 7.08$ & 0.36 \\
\hline
\end{tabular}

a Independent samples $t$ test.

Table 2. Comparison of the Mean Difference Between the Initial Angle, Second Week, and Fourth Week in Both Legs With Active Dynamic Training and Passive Static Training

\begin{tabular}{|c|c|c|c|}
\hline Time & Group & Mean \pm SD & $P$ Value $^{a}$ \\
\hline \multirow{2}{*}{ Difference between the initial angle and the second week } & Active dynamic & $7.37 \pm 5.06$ & $<0.001$ \\
\hline & Passive static group & $2.75 \pm 2.33$ & $<0.001$ \\
\hline \multirow{2}{*}{ Difference between the initial angle and the fourth week } & Active dynamic & $9.25 \pm 5.13$ & $<0.001$ \\
\hline & Passive static group & $3.98 \pm 3.16$ & $<0.001$ \\
\hline
\end{tabular}

a ANOVA repeated measures. 
injuries, back pain, and biomechanical changes in the area. For this reason, in many countries today, exercises to increase flexibility in this muscle group are included in bodybuilding programs.

Many researchers, including Bandy et al, ${ }^{18}$ Chan et al, ${ }^{27}$ Funk et $a l,{ }^{28}$ and Shadmehr et al, ${ }^{29}$ have studied various types of short-term hamstring treatment methods, such as PNF, warm-up exercises, motorized range exercises, eccentric exercises, diathermy exercises, whole-body vibration training, and a variety of stretching exercises. Many studies have compared the effects of static inactivation training with other methods in the treatment of hamstring tightness. ${ }^{10,17,27,28}$ The above-named studies have shown that static inactivity training is more effective in improving muscle tightness than other treatments.

The treatment methods used in the current study were the inactive static method and the proposed dynamic active stretching training. The research done to date on hamstring tightness treatment methods ${ }^{9,30,31}$ indicates that a study comparing these 2 methods is useful, and its results can be used to determine an effective therapeutic method for hamstring tightness. The current study compared the effects of static inactive and dynamic active exercises on hamstring muscle length.

In the present study, both treatment groups showed a significant improvement in hamstring tightness during the treatment period. Both groups demonstrated significant results in increasing the length of the hamstring muscle. Dynamic active training had a more significant effect on the mean difference between the initial angle and at the second and fourth weeks in both legs.

Bandy et al. studied the effects of static stretching and dynamic range of motion training on hamstring muscle flexibility. ${ }^{18}$ Their results suggest that, although both methods increase hamstring flexibility, static stretching was more effective in increasing the flexibility of hamstring muscles compared to dynamic self-stretching, warm-up, and PNF dynamic exercises.

The results of studies on knee extension by Funk et al, ${ }^{28}$ Nelson and Bandy et al ${ }^{17}$ showed that static methods were not significantly different from PNF and eccentric exercises.

In 2017, Coons et $\mathrm{al}^{19}$ compared the effects of 4 weeks of dynamic stretching and standard stretching (static) on hamstring flexibility in a simultaneous polymetric training program in high school volleyball players. They concluded that both dynamic stretching and standard stretching increase range of motion. No difference in gains in range of motion between the stretching groups was observed. This result may be due to the small sample size in each group or to the study population (volleyball players) which differed from the population in the current study (normal subjects).

In 2015, Shaharuddin and Mondam ${ }^{20}$ investigated the effectiveness of static and dynamic stretching during 4 weeks of training on female students. The results showed that static stretching was more effective in increasing
Research Highlights

\section{What Is Already Known?}

Many studies have shown that shortness of hamstring muscles causes various complications. For decades, static stretching has been used as the standard benchmark for training programs, since it has been shown to increase flexibility versus other stretching. On the other, more studies were shown that active stretching had a positive effect on improving hamstrings flexibility.

\section{What This Study Adds?}

The findings of our study demonstrate that active stretching has better results in comparison of static stretching and has a more effect on hamstring muscle length in less time.

hamstring flexibility than dynamic stretching, and it also helps reduce the risk of injury. Such result differs from the results of the current study, possibly due to sample size or because the current study was of the measurement design.

The results of the current study are in line with those of Meroni et $\mathrm{al}^{32}$ who compared the active stretching and static stretching techniques on hamstring flexibility. Active stretching achieved better results in comparison to static stretching and had a greater effect on hamstring muscle length in less time.

\subsection{Limitations}

The current study was limited by a lack of stiffness data, which would allow a better judgment to be made about hamstring extensibility. Furthermore, only girls were evaluated in this study. Another limitation of this study was the lack of precision measuring devices, such as a digital goniometer.

\section{Conclusion}

According to the results of this research, active dynamic training can be considered as a suitable method for increasing flexibility of the hamstring muscle and, consequently, reducing the complications and problems associated with hamstring tightness in female students.

\section{Authors' Contributions}

All authors contributed equally to this study.

\section{Conflict of Interest Disclosures}

All authors declare no conflict of interest.

\section{Ethical Approval}

Obtaining the informed consent of the participants and their voluntary participation were the most important ethical considerations in this study. This study was registered in the Thai Clinical Trials Registry (identifier: TCTR20191001001, https://www.clinicaltrials.in.th). 


\section{Acknowledgments}

The researchers are grateful to all volunteers who participated in this study.

\section{References}

1. Buckthorpe M, Wright S, Bruce-Low S, et al. Recommendations for hamstring injury prevention in elite football: translating research into practice. Br J Sports Med. 2019;53(7):449-456. doi:10.1136/bjsports-2018-099616.

2. Levangie PK, Norkin CC. Joint Structure and Function: A Comprehensive Analysis. Philadelphia, PA: FA Davis Co; 2005.

3. Travell JG, Simons DG. Myofascial Pain and Dysfunction: The Trigger Point Manual. Lippincott Williams \& Wilkins; 1983.

4. Basmajian JV, Wolf SL. Therapeutic Exercise. Baltimore: Williams \& Wilkins; 1990.

5. Lu TW, Chang CF. Biomechanics of human movement and its clinical applications. Kaohsiung J Med Sci. 2012;28(2 Suppl):S13-S25. doi:10.1016/j.kjms.2011.08.004.

6. Hertling D, Kessler RM. Management of Common Musculoskeletal Disorders: Physical Therapy Principles and Methods. Lippincott; 1999.

7. Pooley S, Spendiff O, Allen M, Moir HJ. Static stretching does not enhance recovery in elite youth soccer players. BMJ Open Sport Exerc Med. 2017;3(1):e000202. doi:10.1136/ bmjsem-2016-000202.

8. Ayala F, Sainz de Baranda P, De Ste Croix M, Santonja F. Comparison of active stretching technique in males with normal and limited hamstring flexibility. Phys Ther Sport. 2013;14(2):98-104. doi:10.1016/j.ptsp.2012.03.013.

9. Rani B, Mohanty PP. A comparison between two active stretching techniques on hamstrings flexibility in asymptomatic individuals. J Dent Med Sci. 2015;14(4):12-16.

10. Ahmed AR, Samhan AF. Short term effects of neurodynamic stretching and static stretching techniques on hamstring muscle flexibility in healthy male subjects. Int J Med Res Health Sci. 2016;5(5):36-41.

11. Cochrane DJ, Stannard SR. Acute whole body vibration training increases vertical jump and flexibility performance in elite female field hockey players. Br J Sports Med. 2005;39(11):860865. doi:10.1136/bjsm.2005.019950.

12. Fakhari $z$, Senobari $m$, Jalaie $s$. Prevalence of hamstring and calf mucles shortness. Modern Rehabilitation. 2008;2(1):4146. [Persian].

13. Koli BK, Anap DB. Prevalence and severity of hamstring tightness among college student: a cross sectional study. Int J Clin Biomed Res. 2018;4(2):65-68. doi:10.5455/ ijcbr.2018.42.14.

14. Hamid MS, Ali MR, Yusof A. Interrater and intrarater reliability of the active knee extension (AKE) test among healthy adults. J Phys Ther Sci. 2013;25(8):957-961. doi:10.1589/jpts.25.957.

15. Gajdosik R, Lusin G. Hamstring muscle tightness: reliability of an active-knee-extension test. Phys Ther. 1983;63(7):10851090. doi:10.1093/ptj/63.7.1085.

16. Webright WG, Randolph BJ, Perrin DH. Comparison of nonballistic active knee extension in neural slump position and static stretch techniques on hamstring flexibility. J Orthop Sports Phys Ther. 1997;26(1):7-13. doi:10.2519/jospt.1997.26.1.7.
17. Nelson RT, Bandy WD. Eccentric training and static stretching improve hamstring flexibility of high school males. J Athl Train. 2004;39(3):254-258.

18. Bandy WD, Irion JM, Briggler $M$. The effect of static stretch and dynamic range of motion training on the flexibility of the hamstring muscles. J Orthop Sports Phys Ther. 1998;27(4):295300. doi:10.2519/jospt.1998.27.4.295.

19. Coons JM, Gould CE, Kim JK, Farley RS, Caputo JL. Dynamic stretching is effective as static stretching at increasing flexibility. J Hum Sport Exerc. 2017;12(4):1153-1161. doi:10.14198/ jhse.2017.124.02.

20. Shaharuddin SS, Mondam S. The Effectiveness of Static and Dynamic Stretching on Hamstring Flexibility after 4-Weeks Training to Prevent the Risk of Injuries. Malays J Med Biol Res. 2015;2(3):175-181.

21. Brotzman SB, Manske RC. Clinical Orthopaedic Rehabilitation: An Evidence-Based Approach: Expert Consult. Elsevier Health Sciences; 2011.

22. Kisner C, Colby LA, Borstad J. Therapeutic Exercise: Foundations and Techniques. FA Davis; 2017.

23. Youdas JW, Suman VJ, Garrett TR. Reliability of measurements of lumbar spine sagittal mobility obtained with the flexible curve. J Orthop Sports Phys Ther. 1995;21(1):13-20. doi:10.2519/ jospt.1995.21.1.13.

24. Erkula G, Demirkan F, Kiliç BA, Kiter E. Hamstring shortening in healthy adults. J Back Musculoskelet Rehabil. 2002;16(2):7781. doi:10.3233/bmr-2002-162-305.

25. Shakya NR, Manandhar S. Prevalence of hamstring muscle tightness among undergraduate physiotherapy students of Nepal using passive knee extension angle test. Int J Sci Res Pub. 2018;8(1):182-187.

26. Donatelli RA, Wooden MJ. Orthopaedic Physical Therapy. Elsevier Health Sciences; 2009.

27. Chan SP, Hong Y, Robinson PD. Flexibility and passive resistance of the hamstrings of young adults using two different static stretching protocols. Scand J Med Sci Sports. 2001;11(2):8186. doi:10.1034/j.1600-0838.2001.011002081.x.

28. Funk DC, Swank AM, Mikla BM, Fagan TA, Farr BK. Impact of prior exercise on hamstring flexibility: a comparison of proprioceptive neuromuscular facilitation and static stretching. J Strength Cond Res. 2003;17(3):489-492. doi:10.1519/00124278-200308000-00010.

29. Shadmehr A, Hadian MR, Naiemi SS, Jalaie S. Hamstring flexibility in young women following passive stretch and muscle energy technique. J Back Musculoskelet Rehabil. 2009;22(3):143-148. doi:10.3233/bmr-2009-0227.

30. Nishikawa Y, Aizawa J, Kanemura N, et al. Immediate effect of passive and active stretching on hamstrings flexibility: a single-blinded randomized control trial. J Phys Ther Sci. 2015;27(10):3167-3170. doi:10.1589/jpts.27.3167.

31. Winters MV, Blake CG, Trost JS, et al. Passive versus active stretching of hip flexor muscles in subjects with limited hip extension: a randomized clinical trial. Phys Ther 2004;84(9):800-807. doi:10.1093/ptj/84.9.800.

32. Meroni R, Cerri CG, Lanzarini C, et al. Comparison of active stretching technique and static stretching technique on hamstring flexibility. Clin J Sport Med. 2010;20(1):8-14. doi:10.1097/JSM.0b013e3181c96722. 